\title{
Neuropathic Pain: Unexplored and Significant Relationship With Psoriatic Arthritis and Functional Parameters
}

\author{
Çağrı Ünal ULUTATAR ${ }^{10}$, Fırat ULUTATAR² ${ }^{2}$, Mehmet Tuncay DURUÖZ \\ ${ }^{1}$ Department of Physical Medicine and Rehabilitation, Sancaktepe Şehit Prof. Dr. İlhan Varank Training and Research Hospital, Istanbul, Turkey \\ ${ }^{2}$ Department of Physical Medicine and Rehabilitation, Pain Medicine Division, Çukurova University School of Medicine, Adana, Turkey \\ ${ }^{3}$ Department of Physical Medicine and Rehabilitation, Division of Rheumatology, Marmara University School of Medicine, İstanbul, Turkey
}

\begin{abstract}
Objectives: This study aims to examine the presence of neuropathic pain (NP) in psoriatic arthritis (PsA) and its relationship with functional parameters.

Patients and methods: The study included 50 PsA patients (18 males, 32 females; mean age 50 years; range, 27 to 68 years) diagnosed according to The Classification Criteria for Psoriatic Arthritis. NP was assessed with PainDETECT questionnaire (PDQ). Associations of NP with quality of life (QoL), sleep and fatigue were analyzed by filling out the Psoriatic Arthritis Quality of Life (PsAQoL), Pittsburgh Sleep Quality Index (PSQI), and Multidimensional Assessment of Fatigue (MAF) scales, respectively. Group analysis was performed with independent-samples Mann-Whitney U test and Kruskal-Wallis test. Spearman's rank correlation coefficient (rho) was used for correlations between functional parameters.

Results: Mean disease duration of the PsA patients was $90.2 \pm 90.8$ months]. The number of patients with ambiguous NP was eight ( 3 males, 5 females) while likely NP was detected in 12 patients ( 2 males, 10 females). The median scores of visual analog scale-pain, PSQI, PsAQoL and MAF were significantly higher in patients with NP $(p<0.05)$. The correlations between PDQ and other functional parameters were found moderate-strong calculated as follows: PSQI ( $r h o=0.44, p=0.003), \operatorname{MAF}(r h o=0.42, p=0.005), \operatorname{PsAQoL}(r h o=0.73, p<0.0001)$.

Conclusion: The existence of NP in PsA is not rare and it has a significant relationship with impaired QoL and sleep disturbances. The assessment of NP in PsA patients is necessary to provide a holistic treatment strategy.

Keywords: Neuropathic pain, psoriatic arthritis.
\end{abstract}

Psoriatic arthritis (PsA) is a chronic disabling condition typically characterized by arthritis and psoriasis variably associated with other extraarticular manifestations. It is characterized by a progressive course of synovial inflammation leading to joint destruction that can be effectively treated with disease modifying antirheumatic drugs (DMARDs) and new therapeutics, including biologics. ${ }^{1}$ However, there are still many patients who suffer pain that is either related or unrelated to disease activity causing substantial impairments in physical function and quality of life (QoL). ${ }^{2}$ This suggests that pain in PsA might not be due to only inflammation and subsequent joint damage. Chronic pain can be nociceptive which occurs as a result of tissue damage without sensory nerve damage or neuropathic which is caused by a lesion or dysfunction of the nervous system. Also, chronic pain can occur without somatic background. ${ }^{3}$ The neuropathic pain (NP) symptoms include several abnormal sensations such as tingling, burning, electric shock-like, hyperalgesia and allodynia. The NP is commonly seen in other rheumatic diseases like rheumatoid arthritis (RA), ankylosing

Received: October 05, 2018 Accepted: September 08, 2019 Published online: December 17, 2019

Correspondence: Çagrı Ünal Ulutatar, MD. Sancaktepe Şehit Prof. Dr. İlhan Varank Eğitim ve Araştırma Hastanesi Fiziksel Tıp ve Rehabilitasyon Kliniği, 34785 Sancaktepe, İstanbul, Türkiye. Tel: +90 216 - 6254657 e-mail: drcagriunal1988@hotmail.com

Ulutatar ÇÜ, Ulutatar F, Duruöz MT. Neuropathic Pain: Unexplored and Significant Relationship With Psoriatic Arthritis and Functional Parameters. Arch Rheumatol 2020;35(3):409-415 
spondylitis (AS), and Sjögren's syndrome (SS). ${ }^{3-5}$ The NP can be assessed by standard electrodiagnostic tools, microneurography, laser evoked potentials, functional neuroimaging and outcome measure tools. Visual analog scale (VAS) and painDETECT questionnaire (PDQ) are used commonly in studies to assess NP. Ongoing pain, paroxysmal and evoked pain and the pain intensity can be measured by VAS. ${ }^{6}$ The PDQ is a self-report scale that is used to distinguish the NP from other types of chronic pain without requiring a clinical examination. ${ }^{7}$ The $\mathrm{PDQ}$ displays good psychometric properties in RA, PsA and axial spondyloarthropathies. ${ }^{8}$ The NP can occur in rheumatic diseases due to compression syndromes, comorbidities, vasculitis or drugs used as treatment. Rheumatic patients with NP display low vitality and low mood. ${ }^{9}$ The NP is associated with greater impairments in several QoL domains such as physical, emotional, role functioning, and sleep. Sleep disturbances are commonly associated with the NP.10 Although there are studies about the NP in several rheumatic diseases using PDQ as a measurement tool, to our knowledge, there is no study in the literature that investigates the NP and its relationship with clinical and functional parameters in PSA patients yet. It is important to study the NP in these patients because it could have important implications for PsA treatment strategies. Therefore, in this study, we aimed to examine the presence of NP in PsA and its relationship with functional parameters.

\section{PATIENTS AND METHODS}

This study was conducted at Marmara University Pendik Training and Research Hospital between January 2017 and April 2017 and included 50 PsA patients (18 males, 32 females; mean age 50 years; range, 27 to 68 years) diagnosed according to The Classification Criteria for Psoriatic Arthritis. Exclusion criteria were coexistence of other rheumatic disease, fibromyalgia, disorders commonly causing NP such as diabetes mellitus, chronic renal failure, or chronic liver disease etc. Fibromyalgia was excluded by using the 2016 fibromyalgia diagnostic criteria. ${ }^{11}$ The study protocol was approved by the Marmara University School of Medicine Ethics Committee. A written informed consent was obtained from each patient. The study was conducted in accordance with the principles of the Declaration of Helsinki.

We could not calculate the sample size, since there was no previous reference study. Therefore, post hoc power analysis of this study was performed. Cohen has set out standardized measures of effect size proposing a simple categorization of small, moderate and large effect size.12 A formula based on G*Power version 3.0.10 (Heinrich-Heine-Universität Düsseldorf, Düsseldorf, Germany) was used to calculate post hoc power. ${ }^{13}$ We calculated the power of the study by computing an effect size of 0.80 (large effect) using a significance level of 0.05 . The post hoc power was found to be 0.86 which was good.

The demographic data (age, sex, height, weight, body mass index [BMI]) and clinical parameters (disease duration, type of PsA, active/ latent psoriasis, presence of dactylitis, major organ involvement, erythrocyte sedimentation rate [ESR], and C-reactive protein [CRP]) of the patients were noted. Disease activity score-28 (DAS-28) was noted for disease activity.

Associations of NP with QoL, sleep and fatigue were analyzed by filling out the Psoriatic Arthritis Quality of Life (PsAQoL), Pittsburgh Sleep Quality Index (PSQI), Multidimensional Assessment of Fatigue (MAF), and VAS-pain assessment tools. The NP in PsA patients was assessed with PDQ which consists of seven items which evaluate course pattern of pain and pain radiation. It also has three 0-10 numerical rating scale scores for current, worst, and average pain severity. A total score which ranges between -1 and 38 is calculated. An overall score is categorized as unlikely NP $(<12)$, ambiguous $N P,{ }^{13-18}$ or likely NP $(\geq 19)$. Turkish version of PDQ was used in this study. ${ }^{14}$ The PsAQoL is a unidimensional measure that is developed by using needs-based model. It is a self-administered instrument containing 20 questions, and the participants give true/not true responses (scores 1 and 0 , respectively). The overall score can range from 0 to 20, with a high score representing poor QoL. ${ }^{15}$ The PSQI is a selfreported questionnaire assessing sleep quality and disturbances for the past one month. The sum of scores for the seven components of the PSQI, including sleep duration, sleep disturbance, sleep latency, daytime dysfunction due to sleepiness, 
sleep efficiency, overall sleep quality, and sleep medication use, yields one global score between 0 and $21 .^{16}$ The PSQI global score discriminates "good sleepers" (PSQI total score $\leq 5$ ) from "poor sleepers" (PSQI total score $>5$ ). Turkish version of PSQI was used in this study. ${ }^{17}$ The MAF consists of 16 items and evaluates fatigue using four dimensions: severity, distress, impact on activities of daily living, and timing. Scores range from 0 (no fatigue) to 50 (severe fatigue). ${ }^{18}$ The VAS is commonly used pain scale which is presented as a $10 \mathrm{~cm}$ horizontal line on which the patient's pain intensity is represented by a point between the extremes of "no pain at all" and "worst pain imaginable. ${ }^{19}$ Physical examinations such as manual muscle testing and sensory examination were performed. Also, tender and swollen joints were examined to calculate DAS-28.

\section{Statistical analysis}

Statistical analyses were performed using the IBM SPSS version 20.0 (IBM Corp., Armonk, NY, USA) software. Patients with ambiguous or likely NP were compared to patients without NP.
Assessment of normality was analyzed using the Shapiro-Wilk test. Univariate differences in the median scores of the patients with NP and without NP groups were examined using independent-samples Mann-Whitney $U$ test. The Kruskal-Wallis test was used to compare the three groups of ambiguous NP, likely NP, and non-NP. The Spearman's rank correlation coefficient (rho) was used for the relationship between continuous variables. Correlation coefficients $>0.50,0.35-0.50$ and $<0.35$ were considered strong, moderate and weak, respectively. Quantitative measures were compared using a Chi-square test. $\mathrm{P}$ value $<0.05$ was accepted as statistically significant.

\section{RESULTS}

The mean disease duration was $90.2 \pm 90.8$ months. The numbers of patients with ambiguous and likely NP were eight ( 3 males, 5 females) and 12 (2 males, 10 females), respectively. Median scores with interquartile range (IQR) and minimummaximum values of patient characteristics and the clinical parameters were depicted in Tables 1 and 2 .

\begin{tabular}{|c|c|c|c|c|c|}
\hline & $\mathrm{n}$ & $\%$ & Median & Min-Max & IQR \\
\hline Age (year) & & & 50.5 & $27-68$ & 17 \\
\hline Body mass index $\left(\mathrm{kg} / \mathrm{m}^{2}\right)$ & & & 27.1 & 19.3-36.6 & 5.3 \\
\hline Disease duration (month) & & & 60 & $1-444$ & 84 \\
\hline Disease activity score-28 & & & 2.7 & $1.4-5.7$ & 1.5 \\
\hline Tender joint count & & & 0 & $0-10$ & 2 \\
\hline Swollen joint count & & & 0 & $0-10$ & 0.3 \\
\hline Erythrocyte sedimentation rate & & & 22 & $5-68$ & 18.5 \\
\hline C-reactive protein & & & 3 & $0-30$ & 4 \\
\hline \multicolumn{6}{|l|}{ Clinical type } \\
\hline Asymmetric oligoarthritis & 33 & 66 & & & \\
\hline Symmetric polyarthritis & 11 & 22 & & & \\
\hline Axial disease predominant & 6 & 12 & & & \\
\hline Active psoriasis & 31 & 62 & & & \\
\hline Dactylitis & 5 & 10 & & & \\
\hline Active arthritis & 11 & 22 & & & \\
\hline \multicolumn{6}{|l|}{ Treatment } \\
\hline csDMARDs & 32 & 64 & & & \\
\hline csDMARDs + steroids & 5 & 10 & & & \\
\hline bDMARDs & 13 & 26 & & & \\
\hline
\end{tabular}


Table 2. Descriptives of outcome measures

\begin{tabular}{lccc}
\hline & Median & Min-Max & IQR \\
\hline PDQ & 7 & $0-34$ & 18.25 \\
PsAQoL & 6.5 & $0-20$ & 8.5 \\
PSQI total & 6 & $0-18$ & 9 \\
MAF & 22.08 & $0-45.8$ & 23.5 \\
\hline
\end{tabular}

Min: Minimum; Max: Maximum; IQR: Interquartile range; PDQ: PainDETECT questionnaire; PsAQoL: Psoriatic Arthritis Quality of Life; PSQI: Pittsburgh Sleep Quality Index; MAF: Multidimensional Assessment of Fatigue.

Table 3. Characteristics of psoriatic arthritis patients with (ambiguous and likely) and without neuropathic pain

\begin{tabular}{lccccccc}
\hline & \multicolumn{2}{c}{ Patients with NP } & & \multicolumn{2}{c}{ Patients without NP } & \\
\cline { 2 - 3 } & Median & IQR & & Median & IQR & \\
\hline Age (year) & 53.5 & 17 & & 46.5 & 17 & 0.38 \\
Body mass index $\left(\mathrm{kg} / \mathrm{m}^{2}\right)$ & 23.8 & 5.8 & & 27.8 & 3.8 & 0.21 \\
Disease duration $(\mathrm{month})$ & 60 & 99 & & 48 & 60 & 0.59 \\
DAS-28 & 3.6 & 1.7 & & 2.5 & 0.8 & $<0.0001$ \\
Erythrocyte sedimentation rate $(\mathrm{mm} / \mathrm{h})$ & 23 & 18 & & 21.5 & 20 & 0.86 \\
C-reactive protein $(\mathrm{N}<5 \mathrm{mg} / \mathrm{L})$ & 3 & 3.7 & & 3 & 5 & 0.94 \\
VAS-pain & 8 & 4 & & 2 & 4 & $<0.0001$ \\
PSQI total score & 10 & 7 & & 4 & 9 & 0.002 \\
PsAQoL & 13 & 7.5 & & 4.5 & 7 & 0.01 \\
MAF & 31.5 & 27.2 & & 16.3 & 25 & 0.03 \\
\hline
\end{tabular}

NP: Neuropathic pain; IQR: Interquartile range; DAS-28: Disease activity score; VAS: Visual analog scale; PSQI: Pittsburgh Sleep Quality Index; PsA: Psoriatic arthritis; PsAQoL: Psoriatic Arthritis Quality of Life; MAF: Multidimensional Assessment of Fatigue.

The median scores of VAS-pain, PSQI, PsAQoL and MAF were significantly higher in patients with NP $(p<0.05)$. Patients with higher disease activity had higher PDQ scores $(\mathrm{p}<0.0001)$. Comparison of median scores of patients with and without NP was depicted in Table 3. There was no significant difference in median scores of demographic (age, BMI) and some of the clinical features (disease duration, ESR, CRP) between patients with (ambiguous and likely) and without NP ( $p>0.05)$. The median scores of age in ambiguous, likely, and non-NP patients were 42 (IQR: 17), 59.5 (IQR: 9), and 46.5 (IQR: 17), respectively. The median scores of age in ambiguous, likely, and non-NP patients were compared with each other using the Kruskal-Wallis test which revealed a significant difference $(p=0.002)$. There was no significant difference in the PDQ scores of patients under conventional synthetic DMARDs and biologic treatments $(p=0.426)$.
There were moderate to strong correlations between PDQ and other clinical parameters. The strongest correlation of PDQ was found with VAS-pain (rho=0.72; $\mathrm{p}<0.0001$ ) and PsAQoL

Table 4. Correlation of painDETECT with patient characteristics

\begin{tabular}{lcc}
\hline & Spearman's Rho & $p$ \\
\hline Age & 0.40 & 0.002 \\
Disease duration & 0.17 & 0.11 \\
DAS-28 & 0.55 & $<0.0001$ \\
VAS-pain & 0.72 & $<0.0001$ \\
PSQI total score & 0.44 & 0.003 \\
PsAQoL & 0.73 & $<0.0001$ \\
MAF & 0.42 & 0.005 \\
\hline
\end{tabular}

DAS-28: Disease activity score; VAS: Visual analog scale; PSQI: Pittsburgh Sleep Quality Index; PsAQoL: Psoriatic Arthritis Quality of Life; MAF: Multidimensional Assessment of Fatigue. 
( $r$ ho $=0.73 ; p<0.0001)$. The correlations of PDQ scores with patients' characteristics were shown in Table 4.

\section{DISCUSSION}

High levels of NP were found in PsA patients even though the disease activity was fairly wellcontrolled. The PDQ, which had well-established reliability and validity, was used to determine the NP in PsA patients. ${ }^{8}$ To our knowledge, no previous studies have thoroughly examined the relationships of NP with sociodemographic, clinical, and QoL related factors in PsA patients. We have shown that the PsA patients were suffering not only inflammatory but also neuropathic pain. The PDQ was the major tool which was commonly used to study NP in rheumatic disorders in the literature. The prevalence rate of NP in RA, AS, seropositive SS, and seronegative SS was 33\%, $64 \%, 37 \%$ and $40 \%$, respectively. In another study, a total of $28 \%$ of PsA patients had likely NP which was significantly higher than for patients with RA and AS. ${ }^{5}$ Our data showed that 24\% of the patients had likely NP and $16 \%$ of the patients had ambiguous NP that was similar to the literature.

The PsA patients presenting with NP features had a 10-fold higher risk of having increased levels of VAS-pain. ${ }^{5}$ The PDQ strongly correlated with VAS-pain scores in the current study. Also, PsA patients with NP had higher VAS-pain scores compared to patients without NP which was statistically significant supporting the literature. The NP was associated with worse QoL in accordance with a study in patients with RA. This study have consistently shown that the presence and severity of NP are associated with considerable impairments particularly in physical and mental health domains. ${ }^{4}$ The presence of NP has been found to be associated with greater impairments in several QoL domains in patients with peripheral and central nervous system lesions in a previous study. ${ }^{10}$ The patients with psoriasis have reduced QoL due to psoriasis involving skin extensively. PsA also reduces QoL and functional status and it produces more role limitations than RA due to emotional problems and more bodily pain. ${ }^{20}$ Moreover, PsA patients with NP had worse QoL compared to those without NP. Sleep disturbances are commonly seen in patients with NP and the severity of sleep disturbance is related to pain intensity. Patients with NP are more likely to develop sleep disorders and in turn the NP is exacerbated by the disturbance of sleep, so their relationship is bidirectional. ${ }^{21}$ In PsA patients, sleep disturbances were found to be associated with fatigue and decreased QoL. ${ }^{22}$ Also, studies have proved that patients who reported chronic pain also had more fatigue, poor mood, and generally higher levels of stress and disability. ${ }^{4,23}$ In our study, the PDQ scores were moderately correlated with the scales that assess sleep disturbance and fatigue supporting the literature. As far as we know, the relationship of NP with fatigue has not been investigated yet. During the flares of PsA, fatigue also emerged as another significant burden. It is important to notice how chronic pain may aggravate fatigue, but also how fatigue may worsen pain. Fatigue can be secondary to chronic pain while it can also predict the development of chronic pain. An animal research has also shown that healthy mice demonstrate neuropathic symptoms after fatigue induction. ${ }^{24}$ Therefore, our PsA patients might have experienced neuropathic symptoms due to the fatigue.

In the current study, patients with higher disease activity had higher PDQ scores which were associated with increased neuropathic pain. However, the parameters that were used to calculate disease activity (DAS-28) like tender joint count (TJC), swollen joint count (SJC), ESR and CRP did not differ between patients with and without NP. Also, neuropathic symptoms were not found to be related to disease duration. Similarly, Koop et al. ${ }^{4}$ found that NP in RA patients was not associated with disease duration. On the other hand, RA patients with co-existent fibromyalgia had significantly higher DAS-28 scores mostly due to the TJC and general health scores in the same study. Also, Ito et al. ${ }^{25}$ detected no marked differences in the disease duration between patients with likely and ambiguous NP, and those with unlikely NP supporting our results. In this study, the result that the patients with NP had higher DAS-28 scores in spite of no significant differences in objective inflammatory indices (TJC, SJC, ESR, and CRP) indicates a larger contribution from non-inflammatory factors 
to the observed disease activity scores in the high PDQ-group ( $\geq 19)$. There was no significant difference in the PDQ scores of patients under conventional synthetic DMARDs and biologics. Also, in a recent study with RA patients, no association was found between synthetic and biologic DMARDs in respect to NP. ${ }^{25}$

Our study has several strengths. To the best of our knowledge, this is the first study that evaluates the NP in PsA patients and its relationship with other functional and clinical features. This study combines the PDQ scale with clinical, demographic and measurements of sleep, QoL, and fatigue. This gives us an opportunity to evaluate the origin of pain in PsA and also the effect it has on daily living of patients. Potential limitations of our study are that the number of patients was relatively small and mood disorders were not assessed with other outcome measures. Further studies can be performed with larger sample size and by collecting data on mood disorders in association with NP in PsA patients.

In conclusion, the data from our study suggest that PsA patients have neuropathic symptoms demonstrated by using PDQ. It is important to evaluate and treat NP of PsA patients in the clinical setting even though in state of remission.

\section{Declaration of conflicting interests}

The authors declared no conflicts of interest with respect to the authorship and/or publication of this article.

\section{Funding}

The authors received no financial support for the research and/or authorship of this article.

\section{REFERENCES}

1. Nas K, Kiliç E, Çevik R, Bodur H, Ataman Ş, Ayhan F, et al. Management of Psoriatic Arthritis: Turkish League Against Rheumatism (TLAR) Expert Opinions. Arch Rheumatol 2018;33:108-27.

2. Bergman MJ. Social and economic impact of inflammatory arthritis. Postgrad Med 2006;Spec No:5-11.

3. Segal BM, Pogatchnik B, Henn L, Rudser K, Sivils KM. Pain severity and neuropathic pain symptoms in primary Sjögren's syndrome: a comparison study of seropositive and seronegative Sjögren's syndrome patients. Arthritis Care Res (Hoboken) 2013;65:1291-8.
4. Koop SM, ten Klooster PM, Vonkeman HE, Steunebrink LM, van de Laar MA. Neuropathic-like pain features and cross-sectional associations in rheumatoid arthritis. Arthritis Res Ther 2015;17:237.

5. Rifbjerg-Madsen S, Christensen AW, Christensen R, Hetland ML, Bliddal $\mathrm{H}$, Kristensen LE, et al. Pain and pain mechanisms in patients with inflammatory arthritis: A Danish nationwide cross-sectional DANBIO registry survey. PLoS One 2017;12:e0180014.

6. Cruccu G, Sommer C, Anand P, Attal N, Baron $\mathrm{R}$, Garcia-Larrea L, et al. EFNS guidelines on neuropathic pain assessment: revised 2009. Eur J Neurol 2010;17:1010-8.

7. Bennett MI, Attal N, Backonja MM, Baron $\mathrm{R}$, Bouhassira $\mathrm{D}$, Freynhagen $\mathrm{R}$, et al. Using screening tools to identify neuropathic pain. Pain 2007;127:199-203.

8. Rifbjerg-Madsen S, Wæhrens EE, DanneskioldSamsøe B, Amris K. Psychometric properties of the painDETECT questionnaire in rheumatoid arthritis, psoriatic arthritis and spondyloarthritis: Rasch analysis and test-retest reliability. Health Qual Life Outcomes 2017;15:110.

9. McWilliams DF, Walsh DA. Pain mechanisms in rheumatoid arthritis. Clin Exp Rheumatol 2017;35:94-101.

10. Jensen MP, Chodroff MJ, Dworkin RH. The impact of neuropathic pain on health-related quality of life: review and implications. Neurology 2007;68:1178-82.

11. Wolfe F, Clauw DJ, Fitzcharles MA, Goldenberg DL, Häuser W, Katz RL, et al. 2016 Revisions to the 2010/2011 fibromyalgia diagnostic criteria. Semin Arthritis Rheum 2016;46:319-29.

12. Cohen J. Statistical Power Analysis for the Behavioral Sciences. 2nd ed. Hillsdale, NJ: Erlbaum; 1988.

13. Faul F, Erdfelder E, Lang AG, Buchner A. G*Power 3: a flexible statistical power analysis program for the social, behavioral, and biomedical sciences. Behav Res Methods 2007;39:175-91.

14. Alkan H, Ardic F, Erdogan C, Sahin F, Sarsan A, Findikoglu G. Turkish version of the painDETECT questionnaire in the assessment of neuropathic pain: a validity and reliability study. Pain Med 2013;14:1933-43.

15. McKenna SP, Doward LC, Whalley D, Tennant A, Emery P, Veale DJ. Development of the PsAQoL: a quality of life instrument specific to psoriatic arthritis. Ann Rheum Dis 2004;63:162-9.

16. Buysse DJ, Reynolds CF 3rd, Monk TH, Berman SR, Kupfer DJ. The Pittsburgh Sleep Quality Index: a new instrument for psychiatric practice and research. Psychiatry Res 1989;28:193-213.

17. Agargun MY, Kara H, Anlar O. The validity and reliability of the Pittsburgh Sleep Quality Index. Turk Psikiyatri Derg 1996;7:107-15

18. Yildirim Y, Ergin G. A validity and reliability study of the Turkish Multidimensional Assessment of Fatigue (MAF) scale in chronic musculoskeletal physical 
therapy patients. J Back Musculoskelet Rehabil 2013;26:307-16.

19. Hawker GA, Mian S, Kendzerska T, French M. Measures of adult pain: Visual Analog Scale for Pain (VAS Pain), Numeric Rating Scale for Pain (NRS Pain), McGill Pain Questionnaire (MPQ), Short-Form McGill Pain Questionnaire (SF-MPQ), Chronic Pain Grade Scale (CPGS), Short Form-36 Bodily Pain Scale (SF-36 BPS), and Measure of Intermittent and Constant Osteoarthritis Pain (ICOAP). Arthritis Care Res (Hoboken) 2011;63:S240-52.

20. Boehncke WH, Menter A. Burden of disease: psoriasis and psoriatic arthritis. Am J Clin Dermatol 2013;14:377-88.

21. Cheatle MD, Foster S, Pinkett A, Lesneski M, $\mathrm{Qu} \mathrm{D,} \mathrm{Dhingra} \mathrm{L.} \mathrm{Assessing} \mathrm{and} \mathrm{managing} \mathrm{sleep}$ disturbance in patients with chronic pain. Anesthesiol Clin 2016;34:379-93.

22. Duruöz MT, Erdem D, Gencer K, Ulutatar F, Baklacıoğlu HŞ. Validity and reliability of the Turkish version of the Jenkins Sleep Scale in psoriatic arthritis. Rheumatol Int 2018;38:261-5.

23. Haythornthwaite JA, Hegel MT, Kerns RD. Development of a sleep diary for chronic pain patients. J Pain Symptom Manage 1991;6:65-72.

24. Sluka KA, Rasmussen LA. Fatiguing exercise enhances hyperalgesia to muscle inflammation. Pain 2010;148:188-97.

25. Ito S, Kobayashi D, Murasawa A, Narita I, Nakazono $\mathrm{K}$. An analysis of the neuropathic pain components in rheumatoid arthritis patients. Intern Med 2018;57:479-85. 